\title{
Detection of the Geminga pulsar with the MAGIC telescopes
}

\author{
Marcos López $^{* a}$, Giovanni Ceribella ${ }^{b}$, Thomas Schweizer $^{b}$, Jezabel R. García ${ }^{b}$, \\ Francesco Dazzi ${ }^{c}$, for the MAGIC Collaboration ${ }^{\dagger}$ \\ ${ }^{a}$ IPARCOS Institute, Universidad Complutense de Madrid, Madrid, Spain \\ ${ }^{b}$ Max-Plank-Institute for Physics, Munich, Germany \\ ${ }^{c}$ INAF Roma, Rome, Italy \\ E-mail: marcos@gae.ucm.es, ceribell@mpp.mpg.de, tschweiz@mpp.mpg.de, \\ jezabel@mpp.mpg.de, francesco.dazzi@inaf.it
}

\begin{abstract}
We present the results of the analysis of Geminga pulsar observations with the MAGIC Telescopes conducted between 2017 and 2019. The data were taken using a trigger system specially developed for reducing the energy threshold below $30 \mathrm{GeV}$, dubbed Sum-Trigger-II. The analysis of the data led to the detection of pulsed gamma rays above $20 \mathrm{GeV}$ from the second emission peak of the Geminga pulsar. The measured spectrum joins smoothly with the one measured at lower energies by Fermi-LAT.
\end{abstract}

36th International Cosmic Ray Conference -ICRC2019-

July 24th - August 1st, 2019

Madison, WI, U.S.A.

\footnotetext{
${ }^{*}$ Speaker.

${ }^{\dagger}$ https://magic.mpp.mpg.de
} 


\section{Introduction}

After the discovery of more than 230 gamma-ray pulsars by Fermi-LAT ${ }^{1}$, attention is moving to the search for pulsars at higher energies. Two of the brighter and younger pulsars in the GeV sky, Crab [1,2] and Vela [3], have already been firmly detected in the Very High Energy (VHE) domain by Cherenkov telescopes. The details on the possible mechanism responsible for VHE pulses are still under debate. The most plausible scenarios involve Inverse Compton scattering, unlike the curvature radiation mechanism used to explain the sharp spectral cutoffs observed in the vast majority of Fermi-LAT pulsars. One important question is now whether there are other pulsars emitting up to high energies. To answer this question, MAGIC has focused on the Geminga pulsar.

Geminga is the archetype of the radio-quiet pulsar population and one of the brightest sources in the GeV sky. Its spectrum, as measured by Fermi-LAT, deviates from an exponential cutoff above $10 \mathrm{GeV}$ [4]. This motivated several attempts to detect Geminga, both with VERITAS [5] and MAGIC [6].

\section{Observations and data analysis}

MAGIC consists of a set of two 17-meter diameter telescopes using the Imaging Air Cherenkov Technique. The telescopes are located at an altitude of $2200 \mathrm{~m}$ a.s.l. on the Roque de los Muchachos Observatory, in La Palma island (Spain). With the standard trigger system, MAGIC achieves an energy threshold of $50 \mathrm{GeV}$ [7]. In order to lower this threshold further, MAGIC has developed in recent years an analogue trigger system, referred to as Sum-Trigger-II [8]. This system allows us to reduce the energy threshold by a factor of two, which is crucial for observing sources like pulsars, GRBs or distant AGN.

Observations of the Geminga pulsar were carried out between 2017 and 2019 with the SumTrigger-II. A dedicated analysis pipepline was developed to exploit the low energy threshold trigger [9]. The analysis includes the use of dedicated Monte Carlo simulations of Extensive Air Showers following the trajectory of the source on the sky to properly account for the effect of the geomagnetic field at tens of GeV. MAGIC observations were then combined with more than 10 years of data provided by the Fermi LAT space telescope. Data from both telescopes were phase-folded using the same pulsar ephemeris. The two peaks visible in the Fermi-LAT light curve were fitted to obtain the expected phase signal regions for the MAGIC analysis.

\section{Results}

Observations with MAGIC have led to the first ground-based detection of the Geminga pulsar above $20 \mathrm{GeV}$. MAGIC detects emission only from the second peak, P2, of the Geminga light curve. The derived spectrum for P2 is in agreement with Fermi-LAT in the overlapping energy range, favoring a power-law fit. No evidence of flux variability is found in the MAGIC energy range.

\footnotetext{
${ }^{1}$ https://confluence.slac.stanford.edu/display/GLAMCOG/Public+List+of+LAT-Detected+Gamma-Ray+Pulsars
} 


\section{Acknowledgements}

We would like to thank the Instituto de Astrofísica de Canarias for the excellent working conditions at the Observatorio del Roque de los Muchachos in La Palma. We acknowledge the financial support of the German BMBF and MPG, the Italian INFN and INAF, the Swiss National Fund SNF, the European ERDF, the Spanish MINECO, the Indian Department of Atomic Energy, the Japanese JSPS and MEXT, the Bulgarian Ministry of Education and Science, the Croatian CSF, the Polish MNiSzW, the Academy of Finland and the Brazilian MCTIC, CNPq and FAPERJ.

\section{References}

[1] S. Ansoldi et al. (MAGIC Coll.) Astronomy \& Astrophysics 585 (2016) A133

[2] G. Ceribella et al., In proceedings of ICRC2019

[3] H. Abdalla et al. (H.E.S.S. Coll.), Astronomy \& Astrophysic 620 (2018) A66

[4] A. Abdo (Fermi-LAT Coll.) ApJ, 720 (2010) 272

[5] E. Aliu. et al. (VERITAS Coll.), ApJ 800 (2015) 61

[6] Ahnen et al. (MAGIC Coll.), Astronomy \& Astrophysics, 591 (2016) A138

[7] J. Aleksić et al. (MAGIC Coll.), Astroparticle Physics 72 (2016) 61

[8] J. R. Garcia et al., Status of the new Sum-Trigger system for the MAGIC telescopes, In Proceedings of the 33th International Cosmic Ray Conference, astro-ph.IM/1404.4219

[9] M. Shayduk, Optimized next-neighbor image cleaning method for Cherenkov Telescopes, In Proceedings of the 33th International Cosmic Ray Conference 